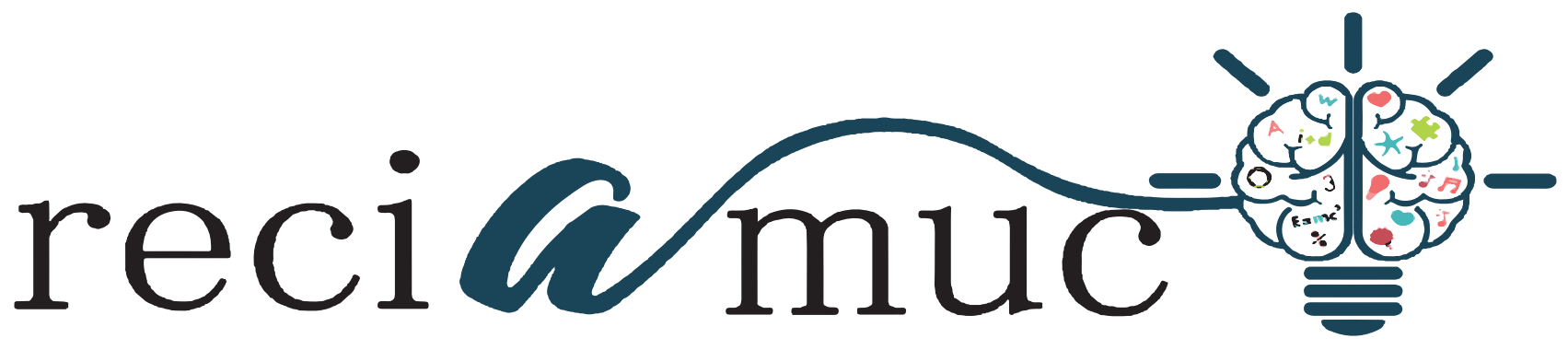

DOI: $10.26820 /$ reciamuc/5.(3).agosto.2021.44-53

URL: https://reciamuc.com/index.php/RECIAMUC/article/view/689

EDITORIAL: Saberes del Conocimiento

REVISTA: RECIAMUC

ISSN: 2588-0748

TIPO DE INVESTIGACIÓN: Artículo de Revisión

CóDIGO UNESCO: 32 Ciencias Médicas

PAGINAS: 44-53

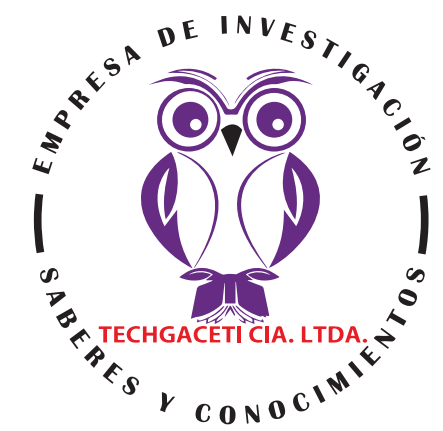

Complicaciones en anestesia raquídea

Complications in spinal anesthesia

Complicações em raquianestesia

\author{
Carolina Alexandra Suárez Ramírez'; Klenger Darío Rosales Vinueza2; \\ Sergio Antonio Barahona Botache3; Michelle Daniela Salamea Saquinaula ${ }^{4}$
}

RECIBIDO: 10/06/2021 ACEPTADO: 12/07/2021 PUBLICADO: 31/08/2021

1. Magister en Salud y Seguridad Ocupacional mención en Prevención de Riesgos Laborales; Medica Cirujana; Investigadora Independiente; Quito, Ecuador; carito140691@gmail.com; (D) https://orcid.org/0000-0003-22265088

2. Médico General de la Universidad Nacional de Chimborazo; Investigador Independiente; Quito, Ecuador; klenger@hotmail.es; (iD https://orcid.org/0000-0001-6204-3004

3. Especialización en Epidemiología y Salud pública; Investigador Independiente; Neiva, Colombia; sergiob91@ hotmail.com; (D) https://orcid.org/0000-0003-3973-2324

4. Médico General; Investigador Independiente; Santiago de Chile; Chile; michelle_salamea@hotmail.com; https://orcid.org/0000-0002-2057-877X

\title{
CORRESPONDENCIA
}

Carolina Alexandra Suárez Ramírez

carito140691@gmail.com

\section{Quito; Ecuador}

() RECIAMUC; Editorial Saberes del Conocimiento, 2021 


\section{RESUMEN}

La anestesia espinal es una herramienta fundamental en la práctica anestesiológica. La anestesia raquídea o espinal (AR/ $\mathrm{AE})$, también conocida como anestesia intradural o intratecal (Al), está asociada al procedimiento médico mediante el cual se requiere una punción en la membrana dura que recubre la médula espinal y, en la columna vertebral inferior, la raíz de los nervios lumbares y sacros (punción lumbar); de la que se extrae una muestra de líquido cefalorraquídeo del canal medular. El uso de la anestesia raquídea puede generar algunas complicaciones o efectos secundarios, los cuales, a pesar de presentarse con poca frecuencia, la gravedad de alguno de estos, lo convierte en un tema de estudio importante para profesionales de la salud y pacientes. En consecuencia, el objetivo del presente estudio consiste en plasmar las complicaciones en anestesia raquídea. Mediante una metodología de revisión, se aportarán: nociones actuales sobre anestesia raquídea, con énfasis en los efectos secundarios o complicaciones. Las principales complicaciones en la anestesia raquídea son: punción hemática, parestesias, náuseas y vómitos, retención urinaria, lumbalgia y cefalea post-punción. Las complicaciones neurológicas en anestesia raquídea son de las más temidas por médicos, pacientes y familiares, entre ellas el daño neurológico y complicaciones cardiovasculares tales como hipotensión, bradicardia o paro cardiaco. Una complicación asociada con la aplicación de los anestésicos en el canal medular es el síndrome neurológico transitorio (SNT), el cual se manifiesta clínicamente por dolor glúteo que se irradia a miembros inferiores. El conocimiento de estas complicaciones o efectos adversos, de sus causas, su prevención y su tratamiento, es imperioso para disminuir, aún más, su incidencia. Asimismo, es importante su estudio para que, en casos de presentarse, la gravedad de sus consecuencias se mínima y evitar daños irreversibles como lo son los daños neurológicos.

Palabras clave: Subaracnoideo, cefalea, bradicardia, técnica anestésica regional, conducto vertebral.

\section{ABSTRACT}

Spinal anesthesia is a fundamental tool in anesthesiological practice. Spinal or spinal anesthesia (RA / AE), also known as spinal or intrathecal $(I A)$ anesthesia, is associated with the medical procedure by which a puncture is required in the hard membrane that covers the spinal cord and, in the lower spine, the root of the lumbar and sacral nerves (lumbar puncture); from which a sample of cerebrospinal fluid is taken from the medullary canal. The use of spinal anesthesia can generate some complications or side effects, which, despite occurring infrequently, the severity of any of these, makes it an important study topic for health professionals and patients. Consequently, the objective of the present study is to capture complications in spinal anesthesia. Through a review methodology, the following will be provided: current notions about spinal anesthesia, with an emphasis on side effects or complications. The main complications in spinal anesthesia are: blood puncture, paresthesia, nausea and vomiting, urinary retention, low back pain and post-puncture headache. Neurological complications in spinal anesthesia are among the most feared by doctors, patients and their families, among them neurological damage and cardiovascular complications such as hypotension, bradycardia or cardiac arrest. A complication associated with the application of anesthetics in the spinal canal is transient neurological syndrome (TNS), which is clinically manifested by gluteal pain that radiates to the lower limbs. The knowledge of these complications or adverse effects, their causes, their prevention and their treatment, is imperative to reduce, even more, their incidence. Likewise, its study is important so that, in cases of occurrence, the severity of its consequences is minimized and irreversible damage such as neurological damage is avoided.

Keywords: Subarachnoid, headache, bradycardia, regional anesthetic technique, spinal canal.

\section{RESUMO}

A raquianestesia é uma ferramenta fundamental na prática anestesiológica. A raquianestesia ou raquianestesia (RA / AE), também conhecida como raquianestesia ou intratecal (IA), está associada ao procedimento médico pelo qual é necessária uma punção na membrana dura que cobre a medula espinhal e, na coluna vertebral, o raiz dos nervos lombar e sacral (punção lombar); do qual uma amostra de líquido cefalorraquidiano é retirada do canal medular. O uso da raquianestesia pode gerar algumas complicações ou efeitos colaterais, que, apesar de ocorrerem com pouca frequência, pela gravidade de qualquer um deles, torna-se um importante tema de estudo para profissionais de saúde e pacientes. Consequentemente, o objetivo do presente estudo é captar as complicações na raquianestesia. Por meio de uma metodologia de revisão, serão fornecidos: noções atuais sobre raquianestesia, com ênfase nos efeitos colaterais ou complicações. As principais complicações na raquianestesia são: punção sanguínea, parestesia, náuseas e vômitos, retenção urinária, lombalgia e cefaleia pós-punção. As complicações neurológicas em raquianestesia estão entre as mais temidas por médicos, pacientes e seus familiares, entre elas os danos neurológicos e as complicações cardiovasculares como hipotensão, bradicardia ou parada cardíaca. Uma complicação associada à aplicação de anestésicos no canal vertebral é a síndrome neurológica transitória (SNT), que se manifesta clinicamente por dor glútea que se irradia para os membros inferiores. O conhecimento dessas complicações ou efeitos adversos, suas causas, sua prevenção e seu tratamento, é imprescindível para reduzir, ainda mais, sua incidência. Da mesma forma, seu estudo é importante para que, nos casos de ocorrência, a gravidade de suas consequências seja minimizada e danos irreversíveis como danos neurológicos sejam evitados.

Palavras-chave: Subaracnoide, cefaleia, bradicardia, técnica anestésica regional, canal vertebral. 
D.

\section{Introducción}

La anestesia espinal es una herramienta fundamental en la práctica anestesiológica. Aunque la primera anestesia regional descrita fue tópica y luego local infiltrativa, la anestesia espinal es la primera técnica publicada de inyección percutánea de anestésico local perineural. La primera descripción de una anestesia espinal planificada fue realizada por el cirujano alemán August Bier en agosto de 1898 para manejo quirúrgico de una lesión de tobillo. Alrededor de la década de 1970 la anestesia espinal tuvo un resurgimiento. Nuevos anestésicos locales, la incorporación definitiva de las agujas punta lápiz, el mejor entendimiento de la fisiología de la anestesia espinal, de sus efectos adversos y su manejo, finalmente han reposicionado esta técnica como segura, con grandes beneficios y una importante alternativa en la anestesia moderna. La anestesia espinal es conocida también como anestesia subaracnoidea, anestesia intratecal, anestesia raquídea o raquianestesia. (De La Cuadra, Altermatt, Kychenthal, Irarrázaval, \& Lacassie, 2021, p. 393, 396)

La anestesia raquídea o espinal (AR/AE), también conocida como anestesia intradural o intratecal $(\mathrm{Al})$, está asociada al procedimiento médico mediante el cual se requiere una punción en la membrana dura que recubre la médula espinal y, en la columna vertebral inferior, la raíz de los nervios lumbares y sacros (punción lumbar); de la que se extrae una muestra de líquido cefalorraquídeo del canal medular. "Durante la anestesia raquídea, se inyecta un medicamento en el conducto vertebral para insensibilizar los nervios de la mitad inferior del cuerpo", es por ello que, una complicación muy común son los dolores de cabeza espinales. (Mayo Clinic, 2021)

Es la administración de un anestésico local en el espacio subaracnoideo o intradural, con el fin de bloquear el estímulo nervioso (sensitivo, autonómico y motor).
Con esta anestesia se consigue un bloqueo secuencial, comenzando por las fibras nerviosas más delgadas (autonómicas y termoalgésicas) y terminando por las más gruesas (tacto, presión y motricidad) (Chasi, 2017)

Por lo general, la anestesia raquídea es usada cuando la intervención es a nivel genital, de las vías urinarias o en las partes inferiores del cuerpo. Sin embargo, es posible que este tipo de anestesia (al igual que en la anestesia epidural) también sea considerada para procedimientos en la zona abdominal; en casos donde la operación o el período de dilatación es demasiado doloroso sin ningún analgésico; cuando es posible dejar el cuerpo del paciente en una posición cómoda durante el procedimiento, y; cuando el paciente solicita que, en lo posible, se evite la menor medicación sistémica y la anestesia general por los propios efectos colaterales que ésta normalmente conlleva. (Enciclopedia Médica A.D.A.M., 2019)

El uso de la anestesia raquídea presenta algunas complicaciones o efectos secundarios, los cuales, a pesar de presentarse con poca frecuencia, la gravedad de alguno de estos, lo convierte en un tema de estudio importante para profesionales de la salud y pacientes. Si bien las bondades y beneficios del uso de este tipo de anestesia supera los riesgos, ningún paciente quiere salir de la sala de operaciones con un daño neurológico permanente.

En consecuencia, al desarrollar presente estudio, se tiene como objetivo aportar un nuevo material bibliográfico que sirva tanto en la actualización de conocimientos como para el fundamento de futuras investigaciones, por ello se ha escogió adelantar una recopilación de varios tipos de recursos bibliográficos contentivos de información pertinente y vigente con los cuales sustentar un abordaje entorno a las complicaciones en anestesia raquídea. Mediante una metodología de revisión, se aportarán: nociones 
actuales sobre anestesia raquídea, efectos secundarios, factores de riesgo y complicaciones asociadas.

\section{Materiales y Métodos}

Tomando en cuenta que el objetivo del presente estudio está centrado en el hallazgo de literatura científica y académica vigente con las cual poder abordar el tema: Complicaciones en anestesia raquídea, se escogió adelantar una investigación de diseño bibliográfico, en el marco de una metodología de revisión bibliográfica.

La búsqueda de los recursos bibliográficos se llevó a cabo a principios de agosto del corriente, mediante el uso de la internet como recurso fundamental, sin menoscabo de las otras formas de investigativas igualmente válidas. Mediante el uso de la referida red global, principalmente se pudo acceder a plataformas digitales caracterizadas como: repositorios institucionales, buscadores especializados y sitios web; destacándose entre los mismos la de la $\mathrm{Bi}$ blioteca Virtual de la Salud (BVS), PubMed, Sciencedirect, Redib, SciELO, Researchgate, Google Académico y otros; en los que se experimentó la búsqueda de la información con base en tres expresiones propias. Estas formulaciones pudieron ser construidas mediante la conjugación de palabras claves y de operadores lógicos y/o booleanos que resultaron en:

1. anestesia raquídea AND Complicaciones; y

2. anestesia raquídea OR anestesia espinal OR anestesia intradural OR anestesia intratecal;

\section{Spinal anesthesia.}

Los resultados obtenidos de dicho proceso fueron considerados suficientes para alcanzar el objetivo propuesto, sin embargo, por la abundancia de los mismos, fue necesaria aplicación determinados criterios de refinamiento a fines de seleccionar definitivamente solo las fuentes y recursos que sirvieran de sustento en la argumentación aquí expuesta. En detalle, ésta depuración respondió al uso, según estuvieran disponibles en cada plataforma utilizada, de determinados filtros que correspondieran a: publicaciones hechas dentro de los últimos 10 años; en español o inglés; con acceso completo (preferiblemente); dentro del área investigativa de Ciencias de la Salud y Medicina Humana; y material bibliográfico concebido como: revisiones sistemáticas, estudios de cohorte, reportes de casos o de controles, ensayos clínicos, guías de práctica clínica; libros y secciones de libros (capítulos y tomos), e-books, protocolos, consensos, manuales, boletines informativos, tesis de grado, posgrado o doctorado, informes, planes y proyectos, entre otras clases de recursos con fundamento científico. También se procuró que, cualquiera de los recursos antes mencionados, estuviesen producidos, avalados o promovidos por instituciones, entes, organizaciones, sociedades o asociaciones de profesionales en el área de la salud o medicina humana, de carácter público o privado, nacionales, internacionales o multilaterales.

Para finalizar este apartado, es importante señalar que se descartó tanto aquel contenido que resultó estar repetido (duplicado) por haberse encontrado en un proceso de búsqueda previo, como también el que se distinguió como editoriales o cartas editoriales, anotaciones académicas y otros tipos de materiales bibliográficos de escaso valor científico, con bajo nivel de evidencia o aportado por tratadistas sin acreditación en el área de ciencias de la salud o medicina humana.

\section{Resultados}

Técnica anestésica regional que consiste en la inyección de anestésicos locales en el espacio subaracnoideo, en el líquido cefalorraquídeo que baña la médula espinal. (...) La anestesia raquídea se utiliza para las intervenciones en las extremidades inferiores, la cadera, el periné, el abdomen bajo 
D.

y la columna lumbar. (Clínica Universidad de Navarra, 2020)

"La anestesia espinal (raquídea) implica la inyección de un medicamento en el canal que rodea la médula espinal. Se utiliza para adormecer el cuerpo por debajo y a veces por encima del sitio de la inyección." (Healthwise, 2021)

\section{J Factores de riesgo}

(1) Entre los riesgos asociados con la anestesia raquídea (paralelamente compartidos con la epidural) están:

- Náusea o dificultad para respirar.

- Daño en los nervios que le provoque adormecimiento crónico o dolor

- Reacción alérgica a la anestesia empleada.

- Sangrado alrededor de la columna vertebral (hematoma).

- Dificultad para orinar.

- Caída en la presión arterial.

- Infección en la columna vertebral (meningitis o absceso).

- Daño neurológico.

- Convulsiones (esto es raro).

- Dolor de cabeza fuerte. (Enciclopedia Médica A.D.A.M., 2019; Drugs, 2021)

\section{Complicaciones y efectos secundarios}

Aunque es raro, las complicaciones que probablemente se asocien con la anestesia regional son: los hematomas, desgarro de estructuras vecinas (ambos a causa de la punción) y la infección en el lugar de la inyección (mayormente relacionada con factores propios en el paciente). Todavía más extrañas, pero más serias, son las complicaciones vinculadas a:

- Las lesiones de los nervios por toxicidad de los anestésicos locales; o también,
- Las que generan un marcado incremento del de hipotensión y bradicardia, que a su vez podría derivar en serios casos de arritmias y excitación o depresión del sistema nervioso central. (MBA Surgical Empowerment, 2018)

Otras complicaciones y efectos secundarios asociados a la AR son:

- Dolor de cabeza. En la Anestesia raquídea se perfora la duramadre y por ese agujero puede salir el líquido céfalo raquídeo, que, al estar en conexión con el cerebro, su falta puede producir dolor de cabeza. Se minimiza el riesgo con agujas finas (agujero más pequeño).

- Dolor de espalda en el sitio donde se ha insertado la aguja.

- Dificultad para orinar, ya que también se afectan los nervios que controlan la micción. (MBA Surgical Empowerment, 2018)

Se hallan coincidencias entre los aportes de Carretero, Martínez, Carretero, \& Juaniz (2011) y Chasi (2017) al agrupar otras de las complicaciones y efectos secundarios de la AR, destacando entre ellos:

- Punción hemática: salida de sangre a través de la aguja.

- Parestesias: sensación desagradable asociada a la punción (hormigueo, calambres...)

- Hipotensión arterial: debida a la vasodilatación provocada por el bloqueo simpático.

- Náuseas y vómitos: secundarios generalmente a la hipotensión.

- Retención urinaria: por bloqueo del músculo detrusor vesical.

- Lumbalgia: sobre todo tras intentos repetidos con agujas de gran calibre.

- Cefalea post-punción: por la continua pérdida de LCR a través del orificio dural. 
La cefalea, es considerada dentro de los efectos secundarios post punción y generalmente es leve, aparece durante las 24-48 h posteriores a la punción, con mayor frecuencia en la región frontal u occipital. Se acentúa en posición erguida y disminuye en decúbito. Puede acompañarse de náuseas, vómitos, mareos, tinnitus, alteraciones visuales y síntomas meníngeos. Cede espontáneamente en un día (a veces después de algunas semanas). Prevención: usar una aguja atraumática, realizar la punción con una aguja más fina (p. ej. $22 \mathrm{G}$ en lugar de $18 \mathrm{G}$ ), direccionar el extremo cortante de la aguja con el bisel hacia la parte lateral de la columna (para que las fibras de la duramadre no sean cortadas, sino separadas). Permanecer en decúbito por más tiempo no previene la cefalea. Tratamiento: reposo en cama, analgésicos VO (paracetamol, paracetamol con cafeína, opioides, en caso de síntomas persistentes parche de sangre autóloga; no utilizar AINE ni fármacos que deterioran la función plaquetaria). (Manual MIBE, 2021)

Las complicaciones neurológicas en anestesia raquídea son de las más temidas por médicos, pacientes y familiares. Las principales complicaciones neurológicas asociadas a los bloqueos neuroaxiales, especialmente la anestesia raquídea, epidural o combinada raquídea-epidural (ARE), son: cefalea pospunción dural (CPPD), daño neurológico y complicaciones cardiovasculares tales como hipotensión, bradicardia o paro cardiaco. Dias Cicarelli, Frerichs, \& Martins Benseñor, (2014) en su estudio para describir la incidencia de la cefalea pospunción dural y daño neurológico en la población obstétrica de un hospital universitario que fue tratada con bloqueo neuroaxial, en comparación con la literatura mundial e identificar los factores de riesgo, llegaron la siguiente conclusión:

Entre las 2399 pacientes analizadas, 7 desarrollaron parestesias, una paciente dolor del nervio ciático y 6 pacientes neuropatía femoral (todas con remisión espontánea después de tratamiento farmacológico). Todas las pacientes que desarrollaron parestesia de las extremidades inferiores se sometieron a bloqueos neuroaxiales y luego se colocaron en posición ginecológica. Este hecho nos hizo asociar la aparición de parestesia con la posición de estas pacientes y no con el bloqueo neuroaxial realizado En nuestro análisis encontramos que el riesgo de parestesia es mayor cuanto más largo es el tiempo en posición ginecológica, pero sin significación estadística. Dicha falta de significación podría deberse a la baja prevalencia de parestesias, lo cual hizo necesario analizar a un mayor número de pacientes. (p. 31)

De la Fuente, Espinoza, \& Altermatt, (2011) en su estudio destacan que es fundamental conocer los mecanismos de daño involucrados y los antecedentes clínicos y complementarios que pueden ayudar a disminuir el riesgo de daños neurológicos. Asimismo, manifiestan de los daños neurológicos en anestesia regional periférica lo siguiente:

Si bien la incidencia de daño neurológico posterior a anestesia regional periférica (DNPAR) es baja, el potencial daño que ella puede ocasionar es relevante[...]Es importante el trabajo en equipo, colaborando con el adecuado posicionamiento del paciente y la participación de cirujanos expertos. El diagnóstico y las intervenciones oportunas son la clave para prevenir resultados neurológicos catastróficos.

El síndrome neurológico transitorio (SNT), fue formalmente descrito por primera vez en 1993 por Schneider, quien reportó cuatro casos de dolor después del uso de lidocaína espinal. El síndrome se manifiesta clínicamente por dolor glúteo que se irradia a miembros inferiores, usualmente, iniciado después de 24 horas de la aplicación de los anestésicos en el canal medular y con una duración máxima de aproximadamente 10 días. (Sará, J., Bernal, González, \& Alzate, 2015, p. 156) 
D.

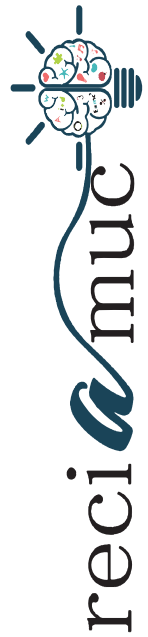

Por su parte, Forget, Borovac, Thackeray, \& Pace, (2019) en cuanto a la frecuencia de los síntomas neurológicos transitorios (SNT) después de la anestesia espinal con lidocaína y compararla con otros tipos de anestésicos locales, destacan:

Los resultados del metanálisis en red y del metanálisis pareado indican que el riesgo de desarrollar SNT después de la anestesia espinal es menor cuando se utiliza bupivacaína, levobupivacaína, prilocaína, procaína y ropivacaína, en comparación con la lidocaína. La administración de 2-cloroprocaína y mepivacaína tuvo un riesgo similar a la lidocaína en cuanto a desarrollar SNT después de la anestesia espinal. Se debe informar a los pacientes sobre los SNT como un posible efecto adverso de la anestesia local con lidocaína, y la elección del agente anestésico se debe basar en el contexto clínico específico y parámetros como la duración esperada del procedimiento y la calidad de la anestesia.

Existen algunas complicaciones raras, entre las que se pueden mencionar:

La paresia de miembros inferiores (causada por hematoma epidural; generalmente en pacientes que recibían tratamiento anticoagulante poco antes o después de la punción); intususcepción de las amígdalas cerebelosas al agujero occipital (en pacientes con edema cerebral, tumor o hemorragia subaracnoidea severa; conduce a la muerte); hemorragia subaracnoidea y subdural; lesión de los ligamentos de la columna vertebral o del periostio vertebral; espondilitis infecciosa; inflamación aguda purulenta de las vértebras, absceso, tumor epidermoide. (Manual MIBE, 2021)

\section{AR en pediatría}

Wiramus, Noël, \& Michel (2017) precisan que, en pediatría, la AR puede ser indicada en función de la cirugía (intervención quirúrgica en los miembros inferiores; en el área abdominal infraumbilical, $y$, raramente, en la zona abdominal supraumbilical) o en ra- zón de las características del paciente (por factores de riesgos o contraindicaciones a la anestesia general). De hecho, en casos de pacientes prematuros (y con edad posconcepcional < 60 semanas) para la cirugía abdominal y de las extremidades inferiores y de una duración inferior a 90 minutos, es reconocida como el «gold standard». Así mismo señalaron que. indistintamente de la razón, este tipo de anestesia comúnmente se efectúa con agujas de punción lumbar que, si bien es cierto que con éstas no es posible evadir por completo el riesgo de tumores dermoides intraespinales, lo recomendable es utilizarlas con el apoyo de un mandril. También aclararon que tales agujas podrían ser de distintos tamaños, su vez relacionados con la edad y/o del peso del niño (ver Tabla 1).

Las complicaciones de la anestesia raquídea en el niño son raras y, en general, menores. La mitad de los efectos secundarios en el estudio de la Association des Anesthésistes-Réanimateurs Pédiatriques d'Expression Française (ADARPEF) se debía a un fallo en la técnica o a un material no apropiado. En la literatura no se encuentra ninguna noción de complicación fatal o de secuela neurológica definitiva. Las complicaciones son las siguientes:

- fracaso del bloqueo inferior al 5\%;

- cefaleas tras punción del conducto raquídeo: muy raras en los niños de menos de 10 años debido a la baja presión del LCR, una duramadre muy elástica y, en el caso de los más pequeños, de la ausencia de posición de pie y de marcha. Sin embargo, la incidencia en los niños entre 2-15 años es próxima a la del adulto, es decir, alrededor del 4-5\%. Los síntomas son en general moderados, con únicamente un 0,1\% de cefaleas graves. El tratamiento es sobre todo sintomático. El parche hemático $(0,2-0,3 \mathrm{ml} / \mathrm{kg})$ sólo debería realizarse si las cefaleas son graves o si persisten más de 1 semana;

- dolor dorsal (5-10\%); 
- $\quad$ anestesia espinal alta o completa (0,6\%): en los casos en los que se elevan las piernas del niño tras la inyección o en caso de sobredosificación;

- síntomas neurológicos transitorios (34\%): dolor y disestesias moderados, que en general irradian desde la región glútea hacia los miembros inferiores;

- toxicidad medular de los fármacos utilizados: toxicidad potencial mayor al inicio de la vida debido a las propiedades dinámicas intrínsecas del desarrollo neuroaxial. También existe un riesgo no despreciable de subestimación de las complicaciones neurológicas para ciertos niños que no son capaces de expresar determinados signos neurológicos o para los que los están aún en edad de caminar;

- infección: únicamente algunos casos clínicos aislados de meningitis (aséptica y séptica). En el caso de fiebre tras punción espinal, está indicada rápidamente una punción lumbar para eliminar este diagnóstico. (Wiramus, Noël, \& Michel, 2017, pág. 7)

Tabla 1. Tamaños de agujas de punción lumbar usadas para AR en pediatría, en función de la edad y/o del peso del paciente.

\begin{tabular}{llcc}
\hline \multicolumn{1}{c}{ Paciente } & Extremo Distal & Tamaño & Longitud \\
\hline Neonato, & Bisel Doble & $26 \mathrm{G}$ & $25-40 \mathrm{~mm}$ \\
lactante & Bisel de Quincke & $22 \mathrm{G}$ & $40-50 \mathrm{~mm}$ \\
Niño & Bisel Doble & $25 \mathrm{G}$ & $50 \mathrm{~mm}$ \\
\multirow{2}{*}{ Adolescente } & Punta Lápiz & $27 \mathrm{G}$ & $80 \mathrm{~mm}$ \\
\hline
\end{tabular}

Nota: Tomado de "Anestesias raquídeas en el niño", de Wiramus, Noël, \& Michel (2017), EMC, 43(1), p.6. (doi: 10.1016/S1280-4703(16)81742-9)

\section{Conclusión}

Es importante destacar de las complicaciones por anestesia raquídea, que su incidencia es muy baja, comparado con los beneficios que dichas técnicas ofrecen, sobre todo en el área de la obstetricia.

Las principales complicaciones en la anestesia raquídea son: punción hemática, parestesias, náuseas y vómitos, retención urinaria, lumbalgia y cefalea post-punción. Las complicaciones neurológicas en anestesia raquídea son de las más temidas por médicos, pacientes y familiares, entre ellas el daño neurológico y complicaciones car- diovasculares, tales como, hipotensión, bradicardia o paro cardiaco.

Una complicación asociada con la aplicación de los anestésicos en el canal medular es el síndrome neurológico transitorio (SNT), el cual se manifiesta clínicamente por dolor glúteo que se irradia a miembros inferiores.

El conocimiento de estas complicaciones o efectos adversos, de sus causas, su prevención y su tratamiento, es imperioso para disminuir, aún más, su incidencia. Asimismo, es importante su estudio para que, en casos de presentarse, la gravedad de sus consecuencias se mínima y evitar daños

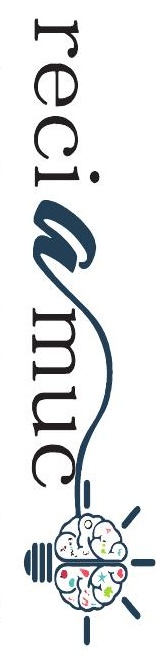


irreversibles como lo son los daños neurológicos.

\section{Bibliografía}

Carretero, S., Martínez, Z.-I., Carretero, E., \& Juaniz, T. (septiembre-octubre de 2011). Anestesia epidural y anestesia raquídea. Zona Hospitlaria [Revista >Electrónica], Anestesiología(31), Unica [publicción eletrónica|]. Recuperado el 20 de Aagosto de 2021, de Sitio web: zonahospitalaria.com: https:// zonahospitalaria.com/anestesia-epidural-y-anestesia-raquidea/

Chasi, H. (2017). Anestesia epidural vs. anestesia raquídea: ¿Qué debes conocer? AEMPPI Ecuador. Recuperado el 20 de agosto de 2021, de https:// www.elsevier.com/es-es/connect/medicina/anestesia-epidural-vs-anestesia-raquidea-que-debes-conocer

Clínica Universidad de Navarra. (2020). Inicio|Actulidad|Diccionario Médico:cun. Obtenido de Sitio web: cun.es: https://www.cun.es/diccionario-medico/terminos/anestesia-raquidea

De La Cuadra, J., Altermatt, F., Kychenthal, L., Irarrázaval, M., \& Lacassie, H. (2021). Anestesia espinal: Parte I. Historia. Revista Chilena de Anestesia, 50, 393-397. doi:10.25237/revchilanestv50n02-16

De la Fuente, N., Espinoza, A., \& Altermatt, F. (2011). Daño neurológico en anestesia regional periférica. Revista Chilena Anestesia, 40, 253-262. Recuperado el 28 de Agosto de 2021, de https://revistachilenadeanestesia.cl/PII/revchilanestv50n02-16. pdf

Dias Cicarelli, D., Frerichs, E., \& Martins Benseñor, F. E. (2014). Incidencia de complicaciones neurológicas y cefalea pospunción dural luego de anestesia regional en la práctica obstétrica: un estudio retrospectivo de 2399 pacientes. Revista Colombiana de Anestesiología, 42(1), 28-32. doi:https:// www. redalyc. org/articulo.oa?id $=195130030006$

Drugs. (02 de agosto de 2021). CareNotes/Anestesia Raquídea: drugs. Recuperado el 20 de agosto de 2021, de Sitio web: drugs.com [en español]: https://www.drugs.com/cg_esp/anestesia-raqu\%C3\%ADdea-ambulatory-care.html

Enciclopedia Médica A.D.A.M. (15 de abril de 2019). Enciclopedia médicalAnestesia raquídea y epidural: MedlinePlus. Recuperado el 20 de agosto de 2021, de Sitio web: medlineplus.gov [en español]: https://medlineplus.gov/spanish/ency/article/007413.htm
Forget, P., Borovac, J. A., Thackeray, E. M., \& Pace, N. L. (2019). Síntomas neurológicos transitorios (SNT) después de la anestesia espinal con lidocaína versus otros anestésicos locales en pacientes adultos quirúrgicos: un metanálisis en red. Cochrane Database of Systematic Reviews, 1-81. doi:https://doi.org/10.1002/14651858.CD003006. pub4

Gonzalo, V., Rivero, M., Pérez, M., López, A., \& Malu$\mathrm{ff}, \mathrm{A}$. (2007). Historia de la raquianestesia y de la anestesia epidural en España. Archivos Españoles de Urología, 60(8), 973-978. Recuperado el 20 de agosto de 2021, de https://scielo.isciii.es/pdf/urol/ v60n8/historia13.pdf

Healthwise. (11 de febrero de 2021). Individuos y familias|Salud y bienestar|Biblioteca del bienestar/Anestesia raquídea: cigna. Recuperado el 20 de agosto de 2021, de Sitio web: cigna.com [en español]: https://www.cigna.com/es-us/individuals-families/health-wellness/hw/anestesia-raqudea-sts 15008

Manual MIBE. (2021). Manual MIBE: empendium. Recuperado el 20 de agosto de 2021, de Sitio web: empendium.com: https://empendium.com/ manualmibe/chapter/B34.IV.24.12.

Mayo Clinic. (29 de julio de 2021). Atención al paciente e informción médicalEnfermedades y afecciones: Mayo Clinic. Recuperado el 20 de agosto de 2021, de Sitio web: mayoclinic.org [en espñol]: https://www.mayoclinic.org/es-es/diseases-conditions/spinal-headaches/symptoms-causes/syc20377913

MBA Surgical Empowerment. (2018). Blog: mba. Recuperado el 20 de agosto de 2021, de Sitio web: mba-eu: https://www.mba.eu/blog/efectos-secundarios-anestesia/

Sará, J., Bernal, V., González, A., \& Alzate, A. (2015). Síndrome neurológico transitorio por anestésicos locales: un caso clínico con bupivacaína. Medicina U.P.B., 34(2), 155-158. Recuperado el 29 de Agosto de 2021, de https://www.redalyc.org/ pdf/1590/159046947008.pdf 


\section{CITAR ESTE ARTICULO:}

Suárez Ramírez, C. A., Rosales Vinueza, K. D., Barahona Botache, S. A., \& Salamea Saquinaula, M. D. (2021). Complicaciones en anestesia raquídea. RECIAMUC, 5(3), 44-53. https://doi.org/10.26820/reciamuc/5.(3).agosto.2021.44-53

Ga 8 BY NC SA

CREATIVE COMMONS RECONOCIMIENTO-NOCOMERCIAL-COMPARTIRIGUAL 4.0. 